\title{
Theoretical prediction of antiferromagnetism in layered perovskite $\mathrm{Sr}_{2} \mathrm{TcO}_{4}$
}

\author{
Alen Horvat, ${ }^{1,{ }^{*}}$ Leonid Pourovskii, ${ }^{2,3,4}$ Markus Aichhorn, ${ }^{5}$ and Jernej Mravlje ${ }^{1}$ \\ ${ }^{1}$ Jožef Stefan Institute, Jamova 39, Ljubljana, Slovenia \\ ${ }^{2}$ Centre de Physique Théorique, École Polytechnique, CNRS, 91128 Palaiseau Cedex, France \\ ${ }^{3}$ Collège de France, 11 place Marcelin Berthelot, 75005 Paris, France \\ ${ }^{4}$ Materials Modeling and Development Laboratory, National University of Science and Technology "MISIS", Moscow, Russia \\ ${ }^{5}$ Institute of Theoretical and Computational Physics, TU Graz, NAWI Graz, Petersgasse 16, Graz, Austria
}

(Received 13 January 2015; revised manuscript received 10 March 2017; published 10 May 2017)

\begin{abstract}
We theoretically investigate the magnetic properties of $\mathrm{Sr}_{2} \mathrm{TcO}_{4}$, a $4 d$ transition-metal layered perovskite of the $\mathrm{K}_{2} \mathrm{NiF}_{4}$ type with half-filled $t_{2 g}$ states. The effect of local Coulomb repulsion between the $t_{2 g}$ orbitals is included within the density-functional theory (DFT) $+U$ and DFT + dynamical mean-field theory (DMFT) methods. The DFT + DMFT predicts paramagnetic $\mathrm{Sr}_{2} \mathrm{TcO}_{4}$ to be close to the Mott insulator-to-metal transition, similarly to the cubic compound $\mathrm{SrTcO}_{3}$. The intersite exchange interactions computed within the DFT + DMFT framework point to a strong antiferromagnetic coupling between the neighboring Tc sites within the layer. We then evaluate the Néel temperature $T_{N}$ within a classical Monte Carlo approach including dipolar interactions, which stabilize the magnetic order in the frustrated $\mathrm{K}_{2} \mathrm{NiF}_{4}$ lattice structure. Our approach is validated by applying it to a set of layered and cubic perovskites, for which we obtain $T_{N}$ in fair agreement with experiment. Within the same approach we obtain the $T_{N}$ of $\mathrm{Sr}_{2} \mathrm{TcO}_{4}$ to be about $450 \mathrm{~K}$. We explore also the effect of anisotropy in exchange interactions due to spin-orbit coupling. These lead to a somewhat higher transition temperature, $550 \mathrm{~K}$.
\end{abstract}

DOI: 10.1103/PhysRevB.95.205115

\section{INTRODUCTION}

Recently, antiferromagnetic (AFM) behavior persisting to high temperatures has been found in the $\mathrm{Tc}^{4+}$ perovskites $\mathrm{SrTcO}_{3}$ and $\mathrm{CaTcO}_{3}[1,2]$. The occurrence of robust magnetism in a $4 d$ compound was not expected, since most $4 d$ oxides are nonmagnetic, in contrast to the more localized $3 d$ oxides. Before this discovery Tc perovskites have not been intensely investigated, the main reason being the radiactivity of Tc. On the other hand, encountering a new class of compounds in a familiar perovskite lattice with little experimental information provides a unique opportunity to test theoretical methods. In this paper we theoretically investigate the magnetic properties of layered perovskite $\mathrm{Sr}_{2} \mathrm{TcO}_{4}$.

Tc perovskites are special among $4 d$ compounds because they have a half-filled $t_{2 g}$ shell [3]. Several theoretical works $[1,4,5]$ have discussed the important role of more itinerant $4 d$ orbitals. The larger spatial extent of $4 d$ orbitals (in comparison to $3 d$ ones) gives rise to a larger hybridization and hence tuneling $t$ and smaller values of the Hubbard repulsion $U$, which in turn enhance the exchange interactions (which follow $t^{2} / U$ for a simple model). Despite smaller values of $U$ the electronic correlations must remain sizable [1,4-8]. If the interactions were too small, no localized magnetic moments would be established. The key ingredient that helps localization is the Hund's rule coupling $J_{H}$ [9], which, for the half-filled shell, increases the cost of charge excitations,

$$
U_{\text {eff }}=E(N+1)+E(N-1)-2 E(N),
$$

with $E(N)$ being the energy of an ion with $N$ electrons [3]. For instance, for a three-orbital Hubbard model $U_{\text {eff }}=U+2 J_{H}$ at half-filling and $U_{\text {eff }}=U-3 J_{H}$ away from half-filling. As a result of the opposite effects of the delocalization due to

\footnotetext{
*alen.horvat@ijs.si
}

the larger extension of $4 d$ orbitals, on one hand, and of the localization due to the Hund's rule coupling, on the other hand, the Tc compounds happen to be situated close to the itinerant-to-localized transition [3]. For magnetism this is very important, and the Néel temperatures are maximal there [5]. Other $4 d$ oxides do not have half-filled shells [9], the localization due to Hund's rule coupling does not apply, and the localized antiferromagnetism occurs only rarely.

A layered $\mathrm{Tc}^{4+}$ perovskite, $\mathrm{Sr}_{2} \mathrm{TcO}_{4}$, has been synthesized too [10]. It crystallizes in the layered body-centered tetragonal $\mathrm{K}_{2} \mathrm{NiF}_{4}$-type lattice, with the lattice constants $a=3.902 \AA$, $c=12.72 \AA$, and space group I4/mmm [10,11]. To our knowledge, only its basic crystal structure has been reported, but more detailed measurements, in particular, determination of the magnetic structure, have not been performed to date.

From the similarity to cubic $\mathrm{SrTcO}_{3}$ one may expect sizable magnetic interactions also in layered $\mathrm{Sr}_{2} \mathrm{TcO}_{4}$ [6], but the question of the long-range ordering in layered systems is more subtle [12], in particular, for lattices of the $\mathrm{K}_{2} \mathrm{NiF}_{4}$-type. These violate the general expectations that in quasi-two dimensions the magnetic susceptibility increases exponentially with diminishing temperature [13] and that hence the magnetic transition is quickly stabilized by any nonvanishing interlayer coupling $J_{\perp}$. More precisely, what one expects is that the transition temperature is only logarithmically suppressed,

$$
T_{c} \sim T_{c}^{3 \mathrm{D}} / \log \left(b J / J_{\perp}\right),
$$

with diminishing $J_{\perp}$ ( $b$ is a model-dependent number) [13-16].

In the present case of the body-centered $\mathrm{K}_{2} \mathrm{NiF}_{4}$ structure [Fig. 1(a)] with checkerboard AFM in-plane magnetic order, the reason this argument fails is because frustration suppresses the effective coupling between layers. Namely, in such an arrangement, depicted in Fig. 1(b), each spin is equally coupled to the same number of oppositely oriented spins in the layer 


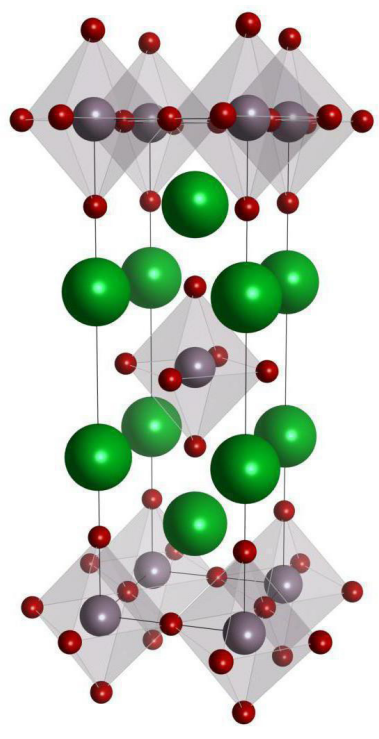

(a)

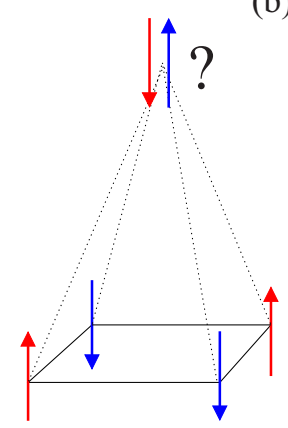

(c)
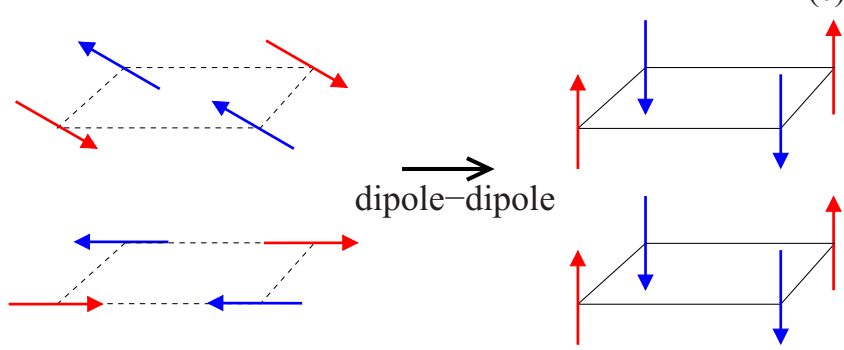

FIG. 1. (a) $\mathrm{Sr}_{2} \mathrm{TcO}_{4}$ crystal structure, visualized using Ref. [22]. Tc ions are represented by gray spheres in the centra of octahedra of oxygen ions. (b) Frustration of the out-of-plane order in the case of the body-centered tetragonal unit cell and in-plane antiferromagnetism. (c) The dipole-dipole interaction energy of the checkerboard antiferromagnetic state is minimized if the moments point out of the plane. Dipole-dipole interactions also couple the layers, as schematically presented.

above (and likewise below), hence the net interplane coupling cancels out.

However, in spite of frustration, the AFM order is experimentally found in lattices of this kind, for instance, in $\mathrm{K}_{2} \mathrm{NiF}_{4}$ [17,18] and $\mathrm{Rb}_{2} \mathrm{MnF}_{4}$ [19]. An important role in stabilizing the magnetic order in such lattices is played by dipole-dipole interactions [17,19-21]. Dipole-dipole interactions have two effects. First, they lower the energy of a state with the magnetic moments pointing out of plane [see Fig. 1(c)] (note that the magnetic moments are enforced to be checkerboard AFM due to the exchange coupling, which is substantially larger than the dipole-dipole interactions). This effect of dipole-dipole interactions is hence similar to that of an easy-axis single-ion anisotropy term $\Delta S_{z}^{2}$ with $\Delta<0$. This kind of anisotropy stabilizes the long-range order already in a single layer, whenever the correlation length becomes long enough. (Once this occurs, the long-range order in three dimensions follows due to any nonvanishing next-nearest-layer coupling.) Second, the dipole-dipole interactions couple the spins in different layers, which helps stabilize the long-range order too. Finally, besides dipole-dipole interactions, the magnetism can also be stabilized by spin-orbit coupling, which leads to anisotropy in exchange interactions.

In the present work we study theoretically the electronic structure and magnetic properties of $\mathrm{Sr}_{2} \mathrm{TcO}_{4}$. We calculate the exchange interactions using a linear-response approach based on the density-functional theory (DFT) + dynamical meanfield theory (DMFT) and find a large AFM coupling between the nearest-neighboring Tc moments. In order to determine the Néel temperature, we employ a classical spin Monte Carlo technique with dipole-dipole interactions included [23,24]. We test our approach on a set of layered and cubic perovskites, for which the experimental values of exchange parameters are known. This allows us to estimate systematic errors of our theoretical exchange interactions. With a correction for this systematic error included, we predict the transition temperature of $\mathrm{Sr}_{2} \mathrm{TcO}_{4}$ to be about $450 \mathrm{~K}$. We calculate also exchange anisotropies due to spin-orbit coupling. We find that these are of the easy-axis kind and nonnegligible $\left(\Delta_{J} / J \sim 0.001\right)$. We explore also the effects of this anisotropy and find that it moderately increases the transition temperature, to $550 \mathrm{~K}$.

The paper is structured as follows. In Sec. II we describe our theoretical approach. In Sec. III we present our DFT $+U$ and DFT + DMFT results, from which we infer the magnetic ordering. There we also report the results of the calculated exchange interactions and transition temperatures for a set of perovskite materials, including the predicted value for $\mathrm{Sr}_{2} \mathrm{TcO}_{4}$. The possible relevance of effects that go beyond our methodology is discussed in Sec. IV, and in Sec. V we give our conclusions. In the Appendix we discuss the dependence of the exchange interaction and the transition temperatures on the Coulomb interaction parameters.

\section{METHODS}

\section{A. DFT $+U$ electronic structure calculation}

We have employed a rotationally invariant $\mathrm{DFT}+U \mathrm{im}-$ plementation of the WIEN2k package [25]. We used $\tilde{U}=$ $U^{S}-J_{H}^{S}=2.0 \mathrm{eV}$ for the Coulomb interaction parameter, compatible with constrained-RPA estimates [26]. $F_{0}, F_{2}$, and $F_{4}$ are the Slater integrals, and $U^{S}=F_{0}$ and $J_{H}^{S}=\left(F_{2}+\right.$ $\left.F_{4}\right) / 14$ are the interaction and Hund's coupling parameters in the Slater notation, respectively. The double-counting correction term [27] was taken in the fully localized limit, and we used the generalized-gradient approximation (GGA) [28] as the approximation to the exchange correlation potential. The Brillouin zone integration was carried out with $1000 \mathbf{k}$ points in the full Brillouin zone, which corresponded to 56 (150) $\mathbf{k}$ points in the tetragonal (orthorhombic) irreducible Brillouin zone. The muffin-tin radii were fixed at 1.81, 2.1, and 1.6 a.u. for Tc, Sr, and O, respectively, in all total energy and structural relaxation calculations.

\section{B. DFT + DMFT electronic structure calculation}

The influence of electronic correlations was investigated within the DFT + DMFT approach too. We used an efficient implementation of this method as provided in the TRIQS package [29-32]. Based on DFT calculations within the 
local-density approximation (LDA) using WIEN2k, we constructed Wannier functions for the $t_{2 g}$ orbitals, which serve as a basis for the DMFT calculations. We solved the DMFT quantum impurity problem using the TRIQS implementation $[33,34]$ of a hybridization-expansion continuous-time quantum Monte Carlo method [35,36], including full rotational invariant interactions [37].

We performed paramagnetic as well as magnetic calculations. For magnetic calculations, we used a doubled unit cell containing two oppositely oriented $\mathrm{Tc}^{4+}$ spins. This cell can accommodate G-type ordering, which is a checkerboard antiferromagnetic pattern in-plane and ferromagnetic (FM) stacking in the $c$ direction. In the paramagnetic calculation, the standard tetragonal unit cell was used and the self-energies were spin-symmetrized after each iteration of the DMFT loop.

\section{Modelization of magnetic properties}

In order to calculate the magnetic transition temperature we first define an effective Hamiltonian associated with the magnetic degrees of freedom. We assume them to be well described by a quantum Heisenberg model,

$$
\mathcal{H}=-\frac{1}{2} \sum_{i \neq j} J_{i j} \hat{\mathbf{S}}_{i} \cdot \hat{\mathbf{S}}_{j}
$$

where $\hat{\mathbf{S}}$ are spin operators and $J_{i j}$ are intersite exchange interactions. We denote $J_{i, j}=J$ for nearest-neighbor interactions.

We approximate the quantum Heisenberg model, Eq. (3), with the classical one:

$$
\mathcal{H}_{\mathrm{c}}=-\frac{1}{2} \sum_{i \neq j} J_{i j} \alpha \mathbf{S}_{i} \cdot \mathbf{S}_{j} .
$$

$\mathbf{S}_{i}$ are three-dimensional vectors of length 1 , and the constant $\alpha=S(S+1)$ keeps track of the length of the quantum spin. Numerical simulations of the quantum Heisenberg model $[16,38,39]$ and comparisons of the results of the calculated transition temperatures using measured exchange interactions validate this way of mapping the quantum model to the classical one for the compounds that are of interest in this study.

For layered perovskites of the $\mathrm{K}_{2} \mathrm{NiF}_{4}$ type, the dipoledipole magnetic interactions are crucial to explain finite Néel temperatures. Therefore, we add to the short-range Heisenberg-type classical Hamiltonian, Eq. (4), a contribution from long-range dipolar interactions. The resulting total Hamiltonian reads

$$
\mathcal{H}^{\prime}=\mathcal{H}_{\mathrm{c}}+\frac{\tilde{\mu} S^{2}}{2} \sum_{i \neq j} r_{i j}^{-3}\left[\mathbf{S}_{i} \cdot \mathbf{S}_{j}-3\left(\mathbf{S}_{i} \cdot \hat{\mathbf{r}}_{i j}\right)\left(\mathbf{S}_{j} \cdot \hat{\mathbf{r}}_{i j}\right)\right]
$$

We denote $\tilde{\mu}=\left(g \mu_{B}\right)^{2} \mu_{0} /(4 \pi)=0.214 \mathrm{meV}^{3}, g \approx 2$ is the Landé factor, $\mu_{B}$ is the Bohr magneton, and $\mu_{0}$ is the permeability constant. The dipolar contribution is proportional to $S^{2}$, since the energy of a spin with a magnetic moment $g \mu_{B} S$ in a dipolar field generated by a spin with the same magnetic moment is proportional to $S^{2}$ [19].

At this point a few words about the validity of our modelization are in order. The classical model naturally neglects several aspects of the full problem. However, we can validate the approach and make sure that we did not omit anything crucial for the considered class of compounds a posteriori by calculating experimentally known transition temperatures. When using the exchange parameters $J$ in Eq. (5) that are determined from experiment, the calculated values indeed agree well with the measured ones. We discuss this validation in detail below in Sec. III. We note that explicit simulations of the two-dimensional quantum Heisenberg model with additional dipole-dipole interactions are an interesting possibility for future work. Such simulations would be very useful in providing an additional validation for the classical approximation to layered frustrated antiferromagnets.

\section{Monte Carlo simulations}

We describe cubic perovskites with the isotropic Heisenberg model, Eq. (4). We implemented two algorithms: the modified Wolff cluster algorithm [24], which is applicable whenever spin frustration is weak, and the Metropolis-Hasting algorithm with over-relaxation [23]. For the data described in the paper, the two algorithms gave consistent results.

For layered $\mathrm{K}_{2} \mathrm{NiF}_{4}$-type perovskites one needs to correctly incorporate dipole-dipole interactions. As discussed in Sec. I, the coupling of the moment in the center of the unit cell to the moments in the corner of the unit cell vanishes due to frustration. Hence we disregard the central ions, and the remaining ions form a simple cubic tetragonal unit cell. Two layers of spins with long-range dipole-dipole interactions, Eq. (5), and with open boundary conditions in the $z$ direction are simulated as in Ref. [19]. Dipole-dipole interactions were included up to the fifth nearest neighbor. We checked that the results do not change if the range of the dipole-dipole interaction or the number of considered layers [40] is increased further.

\section{E. Calculation of exchange interactions}

Starting from late 1970s several methods have been put forward in order to extract effective intersite exchange interactions from $a b$ initio electronic structure calculations. Those methods are based on the Hubbard-Stratonovich transformation [41,42] or on the so-called "force theorem" [4345] (generalized to the case of DFT + DMFT in Refs. [46] and [47]). In order to predict the transition temperature we have evaluated the exchange interactions between transition metal ions' spins employing the recently developed approach described in Ref. [48], which is similar in spirit to those earlier techniques but is particularly tailored to strongly localized Mott insulators. This approach is based on evaluating the linear response in the symmetry-unbroken (paramagnetic) state to simultaneous fluctuations at two neighboring sites. The paramagnetic state is modeled by the DFT + DMFT in conjunction with the Hubbard-I [49] approximation to the DMFT self-energy (DFT $+\mathrm{HubI}$ ). Restricting those fluctuations to the ground-state multiplet $\{\Gamma\}$ of the transition metal $d$ shell, which is, in the present case, labeled with the spin quantum number $S$ only, one can evaluate [48] all the matrix elements $\left\langle\Gamma_{1} \Gamma_{2}\left|\mathcal{H}_{l e}\right| \Gamma_{3} \Gamma_{4}\right\rangle$ of a low-energy spin Hamiltonian between atomic states $|\Gamma\rangle$ corresponding to different values of $S_{z}$ and, hence, extract all intersite interactions $J$. In the 
absence of spin orbit interaction this Hamiltonian is of the isotropic quantum Heisenberg form, (3), while starting from DFT + DMFT calculations with spin orbit one may evaluate also the resulting anisotropies of intersite interactions [see Eq. (6)]. Having thus computed the relevant spin Hamiltonian, one may evaluate the transition temperature as described in Secs. II C and II D.

\section{RESULTS}

\section{A. $\mathrm{DFT}+\boldsymbol{U}$}

We have considered three kinds of magnetic ordering: ferromagnetic ordering as well as A-type and G-type antiferromagnetic ordering. The A-type AFM structure has the tetragonal $P 4 / \mathrm{mmm}$ space group with the Tc moments aligned ferromagnetically within (001) planes and antiferromagnetically between neighboring planes. The G-type structure has a doubled unit cell of the orthorhombic Cmmm space group, with the moments of Tc in the center of the $a b$ face antiparallel to those of $\mathrm{Tc}$ in the vertices.

The calculated total energy of the AFM G-type phase with the experimental lattice parameters [10] is $0.41 \mathrm{eV}$ per formula unit below those of the FM and AFM A-type ones. The total energy of the A-type phase is just $5 \mathrm{meV}$ below the FM one. Because the moments of nearest-neighbor Tc ions are aligned ferromagnetically in the FM and AFM A-type structures, but antiferromagnetically in the G-type AFM one, we conclude that a very strong nearest-neighbor AFM coupling is by far the most significant magnetic interaction in this system. Longer-range interactions are weaker. For example, the second-nearest-neighbor interaction (the one between the Tc moment in the corner and that in the center of the tetragonal cell) gives opposite sign contributions to the energies of the FM and A-type phases that are almost degenerate in the present case.

The G-type AFM phase is clearly the lowest-energy structure among all considered orderings, and its high stabilization energy with respect to the other two phases can hardly be offset by lattice relaxations. Therefore, we have performed a lattice structure optimization for the G-type AFM phase only. We have found that orthorhombic distortions due to G-type ordering are negligible and performed the full optimization of the $a(=b), c$, and internal coordinates. As one may see in Fig. 2 the theoretical equilibrium volume is $3 \%$ larger than the experimental one due to the usual tendency of the GGA towards volume overestimation. The DFT $+U$ equilibrium lattice parameters $a(=b)$ and $c$ of the Cmmm structure are 5.61 and $12.68 \AA$, respectively. There are two distinctive $z_{\mathrm{Sr}}$ and three distinctive $z_{\mathrm{O}}$ in the $\mathrm{Cmmm}$ structure due to symmetry lifting. However, the difference between the optimized values of the corresponding $z$ parameters is very small (below $0.1 \%$ ) and the resulting values are $z_{\mathrm{O}_{1}\left(\mathrm{O}_{2}\right)}=0.162, z_{\mathrm{O}_{3}}=0.50$, and $z_{\mathrm{Sr}_{1}\left(\mathrm{Sr}_{2}\right)}=0.353$. The latter two values are in almost-perfect agreement with experimental data [11]; only $z_{\mathrm{O}_{1}}$ is slightly smaller in our calculation.

Very similar values are obtained already at the nonmagnetic LDA level.

The density of states (DOS) of the G-type structure calculated within the DFT $+U$ (Fig. 3) shows that the Tc

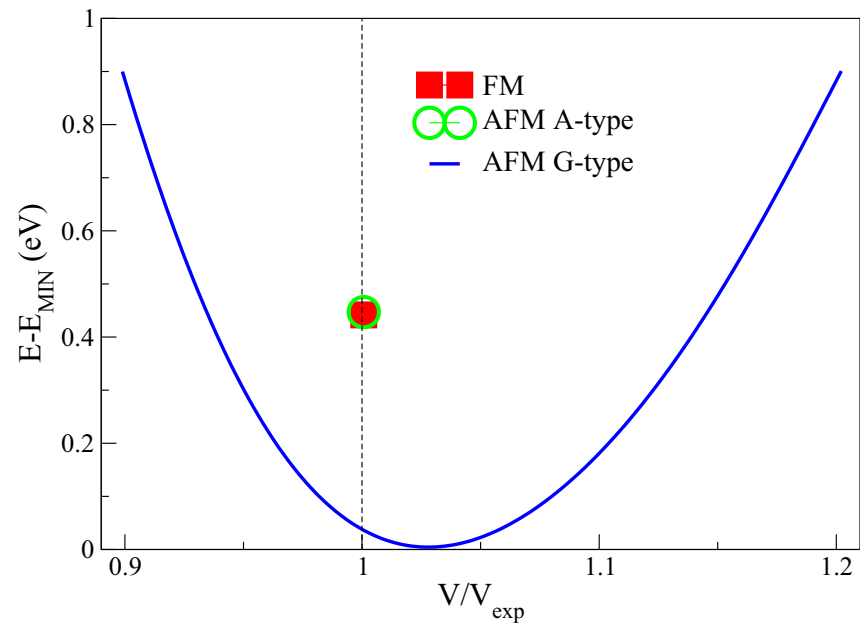

FIG. 2. Solid line: total energy vs volume for the fully relaxed AFM G-type structure of $\mathrm{Sr}_{2} \mathrm{TcO}_{4}$. The square and the circle indicate the total energies of the FM and AFM A-type structures, respectively.

$t_{2 g}$ band is close to full polarization, with almost no occupied $t_{2 g}$ states in the minority channel. As the $t_{2 g}$ states extend also to the oxygen orbitals where the contributions to the spin density in the antiferromagnetic configuration cancel, the moment relevant to neutron measurements can be expected to be smaller. One may try to estimate this moment by looking at the spin polarization within muffin-tin spheres, which gives $1.82 \mu_{B}$. As the $4 d$ atomiclike functions are quite delocalized, some of the spin density extends outside of the muffin-tin sphere. Hence, the above value has to be considered the lower bound on the magnetic moment.

One may also see that the DFT $+U$ gives insulating behavior for $\mathrm{Sr}_{2} \mathrm{TcO}_{4}$, with a gap of about $1.5 \mathrm{eV}$.

\section{B. DMFT results}

For the relaxed structure of $\mathrm{Sr}_{2} \mathrm{TcO}_{4}$ we performed DFT + DMFT calculations. We first enforced the paramagnetic state and investigated the dependence of the electronic structure on the Coulomb interaction parameters. We found

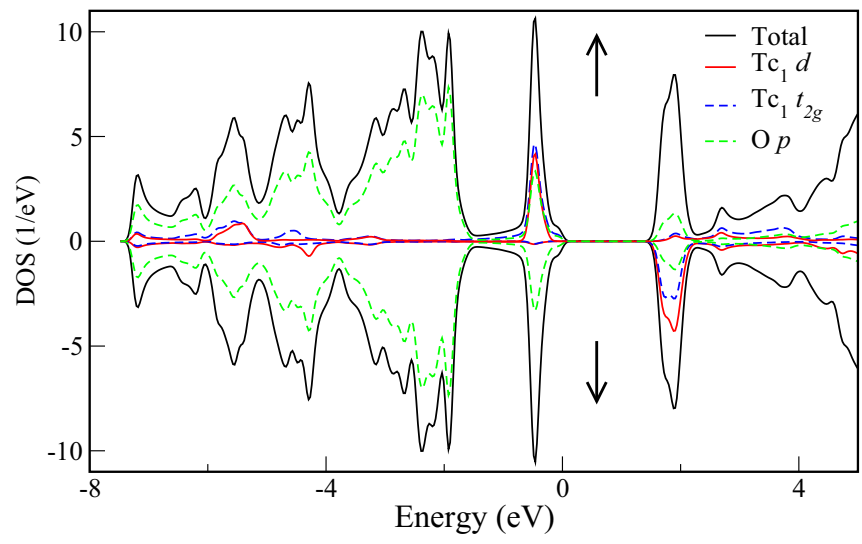

FIG. 3. Total and partial Tc $d, t_{2 g}$, and O $p$ DOS for the G-type AFM structure obtained by the GGA $+U$ method for the theoretical equilibrium lattice structure. 


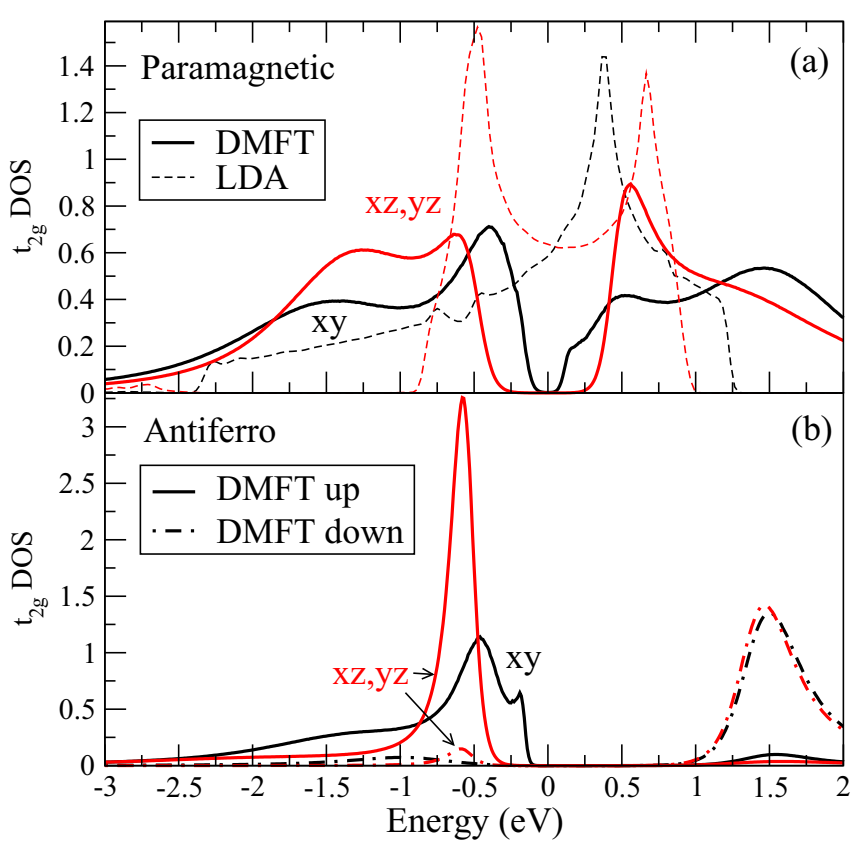

FIG. 4. Orbitally resolved DOS for $t_{2 g}$ states in $\mathrm{Sr}_{2} \mathrm{TcO}_{4}$ calculated from the LDA + DMFT at temperature $T=290 \mathrm{~K}$ and from the LDA. (a) Paramagnetic calculation. (b) Antiferromagnetic calculation.

that the metal-insulator transition in the paramagnetic state occurs at $U_{\text {eff }}=U+2 J_{H}=2.8$, which is slightly lower than the value of 3.0 found for $\mathrm{SrTcO}_{3}$ [5]. The quasitwo-dimensional compound $\mathrm{Sr}_{2} \mathrm{TcO}_{4}$ has a slightly smaller bandwidth, hence the critical interaction for the metal-insulator transition is reduced.

In Fig. 4(a) we plot the orbitally projected local spectral function for $\mathrm{Sr}_{2} \mathrm{TcO}_{4}$ in a paramagnetic state for the parameters $U=2.3, J_{H}=0.3$, which are slightly above the critical value. The stochastic maximum entropy was used for the analytical continuation of quantum Monte Carlo imaginary frequency data [50]. One can observe that the local spectral function is gapped, but that, especially for the $x y$ orbital, the gap is very small, which demonstrates that at these parameters the compound is close to the metal-to-insulator transition. In Fig. 4(a), besides the DMFT spectral function we plot also the orbitally projected LDA DOS in the paramagnetic state. These show that bands spanned by the $x z$ and $y z$ orbitals are narrower, which explains the occurrence of a broader gap in the $x z$ and $y z$ DMFT spectral functions.

We note in passing that stronger correlations in the $x z$ and $y z$ orbitals is opposite to what one finds in the isostructural $4 d^{4}$ compound $\mathrm{Sr}_{2} \mathrm{RuO}_{4}$, where the strongest mass renormalization has been found in the $x y$ orbital, due to its proximity to the van Hove singularity [51]. $\mathrm{Sr}_{2} \mathrm{TcO}_{4}$, being half-filled, is dominated by the proximity to the Mott transition, and the van Hove singularity is further from the Fermi level, which both contribute to the fact that the standard argument applies, which associates the narrower band with stronger correlations.

The fact that the compound is close to the metal-to-insulator transition in the paramagnetic state points to enhanced magnetic properties, as discussed for $\mathrm{SrTcO}_{3}$ [5]. Actually, if in DMFT one allows for the magnetic ordering one gets a
TABLE I. Calculated exchange interactions using our DMFTbased approach $\left(J, J^{\prime}\right)$ compared to the values extracted from $\mathrm{DFT}+U J_{\mathrm{DFT}+U}$ and to the experimental values $J_{\exp }$ from the literature. The unit of exchange interactions is $\mathrm{meV}$. Values of Coulomb interaction parameters at which the $J, J^{\prime}$, and $J_{\mathrm{DFT}+\mathrm{U}}$ are calculated are also listed. Slater conventions $U_{S}=F_{0}, J_{S}=$ $\left(F_{2}+F 4\right) / 14$, and units of $\mathrm{eV}$ are used for these parameters.

\begin{tabular}{lccccc}
\hline \hline Compound & $U_{S}, J_{S}$ & $J$ & $J^{\prime}$ & $J_{\mathrm{DFT}+U}$ & $J_{\text {exp }}$ \\
\hline $\mathrm{KNiF}_{3}$ & $8.0,1.0$ & -12.7 & -0.1 & -7.7 & $-8[52]$ \\
$\mathrm{K}_{2} \mathrm{NiF}_{4}$ & $8.0,1.0$ & -13.5 & -0.1 & -8.4 & $-8.6[52]$ \\
$\mathrm{Rb}_{2} \mathrm{MnF}_{4}$ & $4.0,1.0$ & - & - & -1.1 & $-0.65[52]$ \\
$\mathrm{SrMnO}_{3}$ & $2.5,0.85$ & -8.1 & -0.8 & -9.1 & $-4.14[56]$ \\
$\mathrm{Sr}_{2} \mathrm{MnO}_{4}$ & $2.5,0.85$ & -10.3 & -0.8 & -12.4 & $-6.89[56]$ \\
$\mathrm{SrTcO}_{3}$ & $2.5,0.5$ & -28.2 & -0.9 & -32.8 & - \\
$\mathrm{Sr}_{2} \mathrm{TcO}_{4}$ & $2.5,0.5$ & -35.6 & -0.9 & -45.1 & - \\
\hline \hline
\end{tabular}

behavior very similar to that in the case of $\mathrm{SrTcO}_{3}$ and a similar DMFT ordering temperature, about $2000 \mathrm{~K}$.

In Fig. 4(b) we plot the spin and orbitally resolved DMFT spectral function calculated well in the AFM state at $T=$ 290 K. Compared to Fig. 4(a), one can note that a bigger gap is opened by the onset of magnetic order. The size of the gap is comparable to the one found within the DFT $+U$ (Fig. 3). Conversely, in the pure local spin density approximation the gap in the AFM state is very small, only about $0.05 \mathrm{eV}$.

The high ordering temperatures found in the DMFT calculations should be taken as pointing to strong magnetic correlations but cannot be trusted quantitatively. Furthermore, in quasi-two dimensions these fluctuations become more important than in three dimensions, hence even relative comparison to the values found for $\mathrm{SrTcO}_{3}$ is meaningless.

One can, however, assume that the ordering takes place and calculate from DMFT the value of the ordered magnetic moment at low temperatures. Employing the same scheme as for $\mathrm{SrTcO}_{3}$ [5], i.e., calculating the moment in the set of localized $d$ - $p$ Wannier functions, results in a moment of $2.1 \mu_{B}$. Again, this is significantly smaller than the saturation value of $3 \mu_{B}$. The reason is partly due to covalency and partly due to charge fluctuations, which both arise from the strong hybridization of Tc $d$ with oxygen $p$ states.

To investigate the ordering temperature itself one needs to employ other approaches that are more suitable for quasi-twodimensional systems. We discuss this next.

\section{Transition temperatures within the Monte Carlo approach}

To calculate the transition temperatures we employ Eq. (4) and Eq. (5), and we do simulations within a classical Monte Carlo approach. In order to test the validity of our modelization we calculated the transition temperatures for a set of transitionmetal perovskites for which the values of the transition temperatures and the exchange interactions are known from experiment. The compounds and the corresponding measured exchange interactions $J_{\exp }$ (obtained from neutron and Raman scattering experiments as described in Refs. [52] and [53]) are listed in the first and last columns in Table I.

The critical temperatures are listed in Table II. In the second column we list the experimentally measured values $T_{\exp }$, and 
TABLE II. In column $T_{0}$ we list the calculated critical temperatures for experimental values of exchange interactions; in column $T_{\exp }$, the experimentally measured critical temperatures. $T_{\text {theory }}$ are critical temperatures using theoretical values for the exchange interactions rescaled by 1.6.

\begin{tabular}{lccc}
\hline \hline Compound & $T_{\text {exp }}(\mathrm{K})$ & $T_{0}(\mathrm{~K})$ & $T_{\text {theory }}(\mathrm{K})$ \\
\hline $\mathrm{KNiF}_{3}$ & 253 & 268 & 256 \\
$\mathrm{~K}_{2} \mathrm{NiF}_{4}$ & 97.1 & 105 & 103 \\
$\mathrm{Rb}_{2} \mathrm{MnF}_{4}$ & 38.5 & 40 & - \\
$\mathrm{SrMnO}_{3}$ & 260 & 259 & 231 \\
$\mathrm{Sr}_{2} \mathrm{MnO}_{4}$ & 170 & 162 & 132 \\
$\mathrm{SrTcO}_{3}$ & 1023 & - & 1006 \\
$\mathrm{Sr}_{2} \mathrm{TcO}_{4}$ & - & - & 450 \\
\hline \hline
\end{tabular}

in the third column we list the results of the classical Monte Carlo simulation of Eqs. (4) and (5), using the experimental value $J_{\exp }$ for the nearest-neighbor exchange parameter [54]. In layered perovskites we included the effects of the dipole-dipole interactions as described in Sec. II. We find that the calculated value $T_{0}$ is close to the measured one $T_{\exp }$. This close agreement validates the use of the Heisenberg model with dipole-dipole interactions as well as the quantum-to-classical mapping for the considered class of compounds.

Differently from the materials that we used for validation of the method above, the exchange interactions for Tc perovskites are not known. We therefore calculated the exchange interactions using two approaches: (i) we used the DFT + HubI described in Sec. IIE, and (ii) we also extracted the nearest-neighbor $J_{\mathrm{DFT}+\mathrm{U}}$ from the difference in the GGA $+U$ total energies between FM and AFM structures (we chose AFM cubic and tetragonal structures with all nearest-neighbor transition-metal sites having opposite spin directions). Both approaches depend on the Coulomb interaction parameters that are needed as input. For localized compounds the dependence of the magnetic exchange on the Coulomb interaction parameters is described well by $1 / U_{\text {eff }}$, and it becomes weaker for smaller values of $U_{\text {eff }}$ (see the Appendix). We used values that are close to the ones estimated in the available literature $[26,55]$. The resulting exchange interactions are listed in Table I along with the Coulomb interaction parameters used. In Tc perovksites the exchange interactions are found to be outstandingly large. For $\mathrm{Sr}_{2} \mathrm{TcO}_{4}$ we obtain values even larger than the ones found for $\mathrm{SrTcO}_{3}$.

In Table I we list also the values for the exchange interaction obtained from experiments. Comparing the theoretical values to the measured ones, one notes that the two theoretical approaches behave differently. The DFT $+U$ works well for the localized compounds; for the strongly localized $\mathrm{Ni}$ fluorides the calculated exchange interactions are in close agreement with the experiment. The DFT $+\mathrm{U}$ estimates become progressively worse when the localization becomes weaker and the Mott metal-to-insulator transition is approached. The DFT + HubI approach, on the other hand, gives values that are approximately $60 \%$ larger than the experimental values irrespective of the vicinity of the compound to the Mott transition point [57]. For "ionic" Ni fluorides, less localized $\mathrm{Mn}$ perovskites, and the most itinerant $\mathrm{SrTcO}_{3}$ one observes
TABLE III. Strength of the dipole-dipole coupling (in kelvins) and ratio between dipole-dipole and exchange coupling $v$ normalized to $v\left(\mathrm{Rb}_{2} \mathrm{MnF}_{4}\right)=0.00316$.

\begin{tabular}{lcc}
\hline \hline Compound & $\bar{\mu} S^{2} / a^{3}(\mathrm{~K})$ & $v$ \\
\hline $\mathrm{Rb}_{2} \mathrm{MnF}_{4}$ & 0.2 & 1 \\
$\mathrm{~K}_{2} \mathrm{NiF}_{4}$ & 0.04 & 0.06 \\
$\mathrm{Sr}_{2} \mathrm{MnO}_{4}$ & 0.1 & 0.11 \\
$\mathrm{Sr}_{2} \mathrm{TcO}_{4}$ & 0.1 & 0.03 \\
\hline \hline
\end{tabular}

a similar relative overestimate. As our aim is to predict the transition temperature for a compound that is close to the Mott transition, we employ in the following the DFT + HubI values of $J$ and divide them by 1.6 to correct for the systematic error of our approach.

The critical temperatures $T_{\text {theory }}$ computed within the classical Monte Carlo approach, using the exchange interactions determined in this way, are reported in the last column in Table II. The $T_{\text {theory }}$ values agree reasonably (with errors up to $30 \%$ ) with the measured ones. As a prediction of the transition temperature of $\mathrm{Sr}_{2} \mathrm{TcO}_{4}$ we get a result of $450 \mathrm{~K}$.

\section{DISCUSSION OF EFFECTS OF SPIN-ORBIT COUPLING AND ORTHOROMBIC DISTORTION}

It is interesting to discuss the importance of dipoledipole interactions from a more qualitative perspective. Assuming that the ratio of $c$ and $a$ lattice parameters is constant, one can introduce a dimensionless parameter, $v=\left(\tilde{\mu} S^{2} / a^{3}\right) /\left[J_{\exp } S(S+1)\right]$, that characterizes the ratio of dipole-dipole coupling and exchange coupling strengths (in the case of $\mathrm{Sr}_{2} \mathrm{TcO}_{4}$ for $J_{\exp }$ we take its theoretical value rescaled by 1.6). The value of the parameter $v$ relative to the one found in $\mathrm{Rb}_{2} \mathrm{MnF}_{4}$ is reported in Table III.

The ratio between dipole-dipole interaction and the Heisenberg exchange is lowest in the case of $\mathrm{Sr}_{2} \mathrm{TcO}_{4}$. The transition temperature for a Heisenberg model with nearest-neighbor exchange and dipole-dipole interaction of strength $v$ is shown in Fig. 5.

The discussion has so far ignored the effects of spin-orbit coupling. These lead to the anisotropy of the exchange

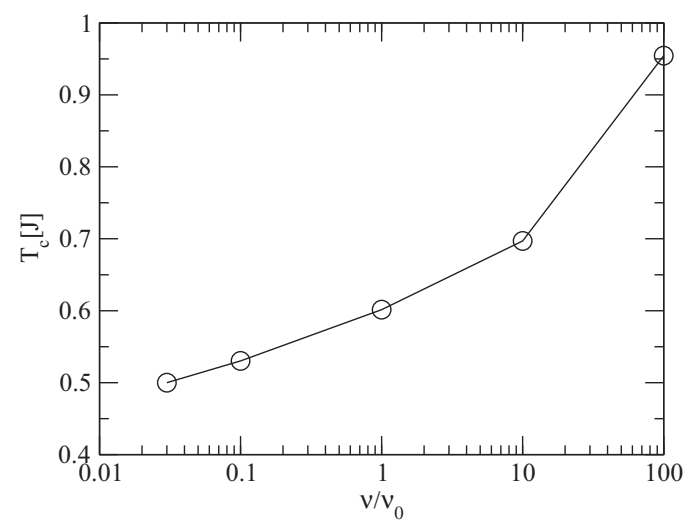

FIG. 5. Transition temperature vs relative dipole-dipole strength $v$ (normalized to the value in $\mathrm{Rb}_{2} \mathrm{MnF}_{4}$ ). 
interactions in the Heisenberg model, Eq. (3), that takes the form

$$
\mathcal{H}=-\frac{1}{2} \sum_{\alpha \in\{x, y, z\}} \sum_{i \neq j} J_{i j}^{\alpha} \hat{S}_{i}^{\alpha} \hat{S}_{j}^{\alpha},
$$

with, in general, unequal components of the exchange interaction $J^{x} \neq J^{y} \neq J^{z}$. We performed also calculation of the exchange interaction starting from the LDA calculation with spin-orbit coupling included. For $\mathrm{Sr}_{2} \mathrm{TcO}_{4}$ this calculation gives $J_{z}$, which is about $0.04 \mathrm{meV}$ larger than the average $J_{x}$ and $J_{y}$ [58], hence we estimate the anisotropy to be about 0.001 and of the easy-axis type. The anisotropies due to the dipole-dipole interaction and due to the spin anisotropy thus work in the same direction, hence one can expect the transition temperature to be raised. Quantitatively, if we include the effect of the anisotropy and set the dipole-dipole interactions to 0 , we obtain a transition temperature of $\mathrm{Sr}_{2} \mathrm{TcO}_{4} T_{N}=$ $550 \mathrm{~K}$ [59], a somewhat larger value than the result for dipole-dipole interactions. This suggests that $\mathrm{Sr}_{2} \mathrm{TcO}_{4}$ is in a regime dominated by the exchange anisotropy due to the relatively low value of dipole-dipole strength and large spin-orbit coupling. Adding the dipole dipole interactions to the calculation with exchange anisotropy included does not lead to a further increase in the transition temperature to our precision.

The orthorhombic distortion (which has not been reported experimentally so far and, hence, is not included in this study) might lead also to a significant reduction in the frustration of the magnetic couplings between layers. This effect would make the magnetic transition more of the $\mathrm{La}_{2} \mathrm{CuO}_{4}$ kind [60] and would increase the transition temperature further. Our DFT $+U$ simulations (Sec. III A) predict that sizable orthorhombic distortions do not occur. If such distortions were realized in the real structure nevertheless, then our results should be taken as an estimate of the lower bound of the transition temperature.

\section{CONCLUSIONS}

In summary, using a combination of theoretical methods that we tested on a set of cubic and layered perovskites, we investigated the properties of the single-layer technetium perovskite $\mathrm{Sr}_{2} \mathrm{TcO}_{4}$. The calculated in-plane exchange interactions are antiferromagnetic and large, which establishes $\mathrm{Sr}_{2} \mathrm{TcO}_{4}$ as a strong two-dimensional antiferromagnet. The body-centered tetragonal unit cell causes frustration: longrange order at the level of the isotropic Heisenberg model does not occur.

We explored the effects of long-range dipole-dipole interactions. The dipole-dipole interactions orient moments perpendicular to the planes and couple the moments in different layers, which stabilizes the magnetic order. This leads to the ordering temperature of about $450 \mathrm{~K}$. We calculated also the anisotropy of the exchange interactions due to relativistic effects, which is found to be of the easy-axis kind, with $J_{x}=$ $J_{y}<J_{z}$, at $\left(J_{x}-J_{z}\right) / J_{z} \approx 0.001$. Including this anisotropy leads to a transition temperature of $550 \mathrm{~K}$.

We note that it would be interesting to dope this compound. Namely, $\mathrm{Sr}_{2} \mathrm{TcO}_{4}$ is, according to our predictions, a strong antiferromagnet, but the magnetic order must disappear upon doping with electrons, as $\mathrm{Sr}_{2} \mathrm{RuO}_{4}$ is known to be a (low-spin) paramagnet that becomes an unconventional superconductor below $1.5 \mathrm{~K}$. The same would happen upon doping with holes, as $\mathrm{SrMoO}_{3}$ is paramagnetic too.

In this respect, not only investigations on layered $\mathrm{Tc}$ compounds [11] but also attempts at material synthesis of other half-filled $4 \mathrm{~d} t_{2 g}$ shell perovskites [61,62] are interesting.

\section{ACKNOWLEDGMENTS}

We warmly thank Antoine Georges for stimulating and helpful discussions. L.P. acknowledges the financial support of the Ministry of Education and Science of the Russian Federation in the framework of the Increase Competitiveness Program of National University of Science and Technology "MISIS" (Grant No. K2-2016-013) as well as program ECOS-MINCyT France-Argentina (Project No. A13E04). M.A. was supported by Austrian Science Fund FWF Projects No. F04103 and No. Y746. J.M. and A.H. acknowledge support from the Slovenian Research Agency under Program No. P1-0044.

\section{APPENDIX: COULOMB INTERACTION PARAMETERS}

\section{Interaction parameters in the Slater and Kanamori conventions}

The Coulomb interaction parameters are usually given in two conventions, the Slater convention or the Kanamori one. For the convenience of the reader in Table IV we report a translation between the two conventions for the parameters relevant to the discussion in the text.

\section{Dependence of exchange interactions in the DFT + HubI approach on Coulomb interaction parameters}

The exchange interactions from the DFT + HubI approach depend on the Coulomb interaction parameters. It is convenient to plot the results as a function of the effective repulsion strength $U_{\text {eff }}=E(N+1)+E(N-1)-2 E(N)$, where $E(N)$ denotes the energy of an ion with $N$ electrons. For a $t_{2 g}$ atom at half-filling this effective interaction is [9] $U_{\text {eff }}=U+2 J_{H}$. We plot the resulting exchange interactions

TABLE IV. Values (in eV) of interaction parameters in Slater notation $U_{S}=F_{0}$ and $J_{S}=\left(F_{2}+F_{4}\right) / 14$ and in Kanamori notation $U$ and $J_{H}$.

\begin{tabular}{lllll}
\hline \hline Compound & $F_{0}$ & $J_{S}$ & $U$ & $J_{H}$ \\
\hline $\mathrm{KNiF}_{3}$ & 8.0 & 1.0 & 9.1 & 0.7 \\
$\mathrm{~K}_{2} \mathrm{NiF}_{4}$ & 8.0 & 1.0 & 9.1 & 0.7 \\
$\mathrm{Rb}_{2} \mathrm{MnF}_{4}$ & 4.0 & 1.0 & 5.1 & 0.7 \\
$\mathrm{SrMnO}_{3}$ & 2.5 & 0.85 & 3.5 & 0.6 \\
$\mathrm{Sr}_{2} \mathrm{MnO}_{4}$ & 2.5 & 0.85 & 3.5 & 0.6 \\
$\mathrm{SrTcO}_{3}$ & 2.5 & 0.5 & 3.07 & 0.38 \\
$\mathrm{Sr}_{2} \mathrm{TcO}_{4}$ & 2.5 & 0.5 & 3.07 & 0.38 \\
\hline \hline
\end{tabular}




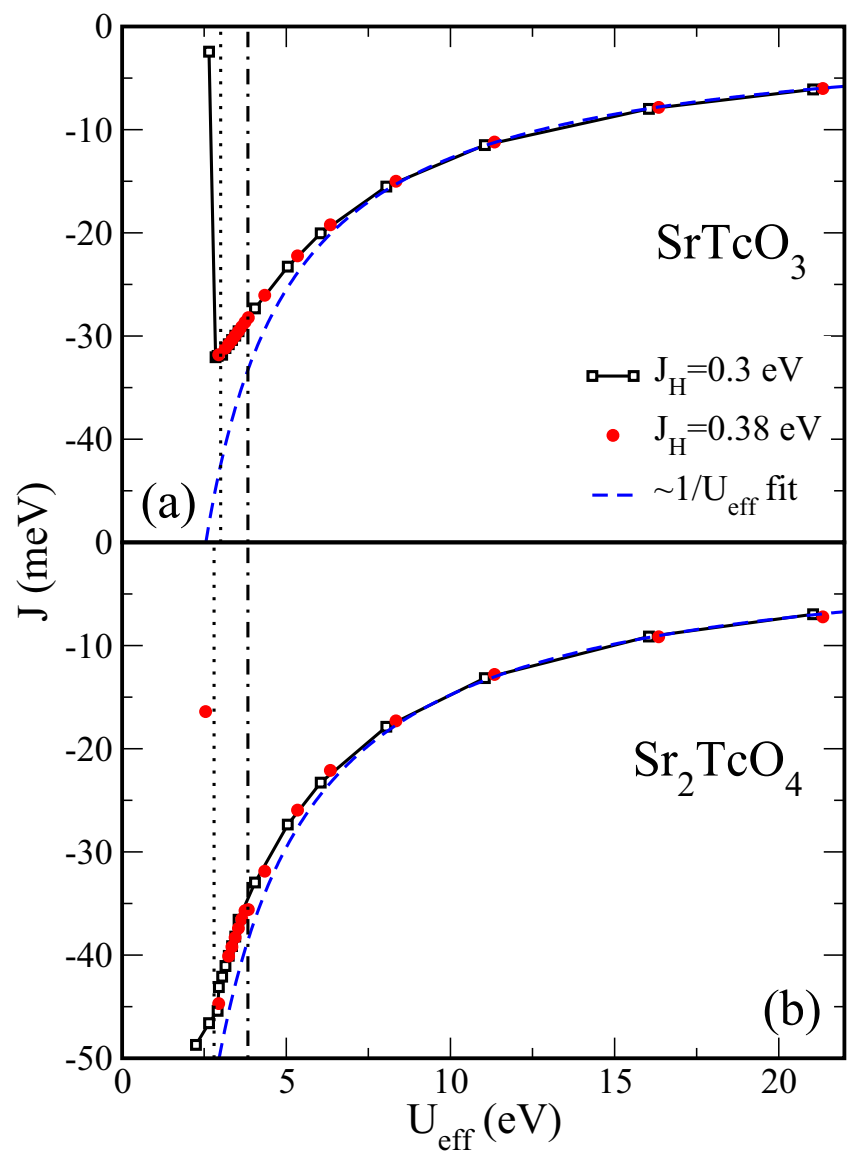

FIG. 6. Dependence of the DFT + HubI exchange interactions on the atomic effective repulsion $U_{\text {eff }}=U+2 J_{H}$ for (a) $\mathrm{SrTcO}_{3}$ and (b) $\mathrm{Sr}_{2} \mathrm{TcO}_{4}$. Vertical dotted line denotes $U_{\text {eff }}$ at which the Mott transition happens. The dashed-dotted vertical line denotes the values of parameters used for prediction of the transition temperature. for $\mathrm{SrTcO}_{3}$ and $\mathrm{Sr}_{2} \mathrm{TcO}_{4}$ in Fig. 6 for two values of $J_{H}$. The interaction values at which the Mott transition occurs in the paramagnetic state is denoted by dotted vertical lines. The interaction value at which we estimated the exchange interaction that we use in the text is denoted by dashed-dotted vertical lines. At large values of the interaction one finds that the dependence follows the localized estimate well, where one expects the exchange interactions to be given by $t^{2} / U_{\text {eff. The }}$ dependence becomes flatter as the metal-to-insulator transition is approached with diminishing $U_{\text {eff }}$. The relative error in the calculated exchange interactions is thus somewhat smaller than the relative error in the effective interaction; for instance, reducing the parameter $U_{\text {eff }}$ from 3.6 to $3 \mathrm{eV}$ will increase the $J$ of $\mathrm{SrTcO}_{3}$ by about $9 \%$ and that of $\mathrm{Sr}_{2} \mathrm{TcO}_{4}$ by about $17 \%$ (and, accordingly, the corresponding magnetic transition temperatures, which are proportional to $J$ ).

Note that although the presence of dipole-dipole interactions (or exchange anisotropy due to spin-orbit coupling) is essential for stabilizing the magnetic order, slight modification of these interactions does not change the transition temperature importantly. We checked that increasing the dipole-dipole interaction strength by a factor of 10 increases the transition temperature by less than $30 \%$ (cf. Fig. 5). Hence the relative change in the Neel temperature is dominated by the relative change in exchange interactions even if the dipole-dipole interactions play an important role.

Below a certain value of $U_{\text {eff }}$, the description of the electronic structure within the DFT + HubI is not reliable anymore, and the approach breaks down. Below this threshold value the nearest-neighbor exchange parameters abruptly drop in a discontinuous way. The fact that this regime is unphysical is reflected also in the fact that the exchange parameters for neighbors that are farther away become larger than the nearest-neighbor ones.
[1] E. E. Rodriguez, F. Poineau, A. Llobet, B. J. Kennedy, M. Avdeev, G. J. Thorogood, M. L. Carter, R. Seshadri, D. J. Singh, and A. K. Cheetham, Phys. Rev. Lett. 106, 067201 (2011).

[2] M. Avdeev, G. J. Thorogood, M. L. Carter, B. J. Kennedy, J. Ting, D. J. Singh, and K. S. Wallwork, J. Am. Chem. Soc. 133, 1654 (2011).

[3] L. de' Medici, J. Mravlje, and A. Georges, Phys. Rev. Lett. 107, 256401 (2011).

[4] C. Franchini, T. Archer, J. He, X.-Q. Chen, A. Filippetti, and S. Sanvito, Phys. Rev. B 83, 220402 (2011).

[5] J. Mravlje, M. Aichhorn, and A. Georges, Phys. Rev. Lett. 108, 197202 (2012).

[6] S. Middey, A. K. Nandy, S. K. Pandey, P. Mahadevan, and D. D. Sarma, Phys. Rev. B 86, 104406 (2012).

[7] G. Wang, L. Li, C. Liu, M. Zhang, and Z. Yang, Phys. Lett. A 376, 3313 (2012).

[8] C.-M. Dai and C.-L. Ma, Mod. Phys. Lett. 28, 1450049 (2014).

[9] A. Georges, L. de'Medici, and J. Mravlje, Annu. Rev. Condens. Matter Phys. 4, 137 (2013).
[10] W. Pies and A. Weiss, in Key Elements: d4-d8-Elements, edited by K.-H. Hellwege and A. M. Hellwege (Springer, Berlin, Heidelberg, 1977), pp. 500-508.

[11] T. Hartmann, A. J. Alaniz, and D. J. Antonio, Proc. Chem. 7, 622 (2012).

[12] N. D. Mermin and H. Wagner, Phys. Rev. Lett. 17, 1133 (1966).

[13] S. Chakravarty, B. I. Halperin, and D. R. Nelson, Phys. Rev. B 39, 2344 (1989).

[14] D. J. Scalapino, Y. Imry, and P. Pincus, Phys. Rev. B 11, 2042 (1975).

[15] H. J. Schulz, Phys. Rev. Lett. 77, 2790 (1996).

[16] C. Yasuda, S. Todo, K. Hukushima, F. Alet, M. Keller, M. Troyer, and H. Takayama, Phys. Rev. Lett. 94, 217201 (2005).

[17] M. Lines, Phys. Rev. 164, 736 (1967).

[18] R. Birgeneau, H. Guggenheim, and G. Shirane, Phys. Rev. B 1, 2211 (1970).

[19] C. Zhou, D. P. Landau, and T. C. Schulthess, Phys. Rev. B 76, 024433 (2007).

[20] R. A. Cowley, G. Shirane, R. J. Birgeneau, and H. J. Guggenheim, Phys. Rev. B 15, 4292 (1977). 
[21] R. J. Christianson, R. L. Leheny, R. J. Birgeneau, and R. W. Erwin, Phys. Rev. B 63, 140401 (2001).

[22] K. Momma and F. Izumi, J. Appl. Crystallogr. 44, 1272 (2011).

[23] D. P. Landau and K. Binder, A Guide to Monte Carlo Simulations in Statistical Physics (Cambridge University Press, Cambridge, UK, 2009).

[24] U. K. Rößler, Phys. Rev. B 59, 13577 (1999).

[25] P. Blaha, K. Schwarz, G. Madsen, D. Kvasnicka, and J. Luitz, WIEN2k, An Augmented Plane Wave + Local Orbitals Program for Calculating Crystal Properties (Techn. Universitat Wien, Wien, Austria, 2001).

[26] L. Vaugier, H. Jiang, and S. Biermann, Phys. Rev. B 86, 165105 (2012).

[27] V. I. Anisimov, I. V. Solovyev, M. A. Korotin, M. T. Czyżyk, and G. A. Sawatzky, Phys. Rev. B 48, 16929 (1993).

[28] J. P. Perdew, K. Burke, and M. Ernzerhof, Phys. Rev. Lett. 77, 3865 (1996).

[29] O. Parcollet, M. Ferrero, T. Ayral, H. Hafermann, I. Krivenko, L. Messio, and P. Seth, Comput. Phys. Commun. 196, 398 (2015).

[30] M. Aichhorn, L. Pourovskii, P. Seth, V. Vildosola, M. Zingl, O. E. Peil, X. Deng, J. Mravlje, G. J. Kraberger, C. Martins, M. Ferrero, and O. Parcollet, Comput. Phys. Commun. 204, 200 (2016).

[31] M. Aichhorn, L. Pourovskii, V. Vildosola, M. Ferrero, O. Parcollet, T. Miyake, A. Georges, and S. Biermann, Phys. Rev. B 80, 085101 (2009).

[32] M. Aichhorn, L. Pourovskii, and A. Georges, Phys. Rev. B 84, 054529 (2011).

[33] P. Seth, I. Krivenko, M. Ferrero, and O. Parcollet, Comput. Phys. Commun. 200, 274 (2016).

[34] L. Boehnke, H. Hafermann, M. Ferrero, F. Lechermann, and O. Parcollet, Phys. Rev. B 84, 075145 (2011).

[35] E. Gull, A. J. Millis, A. I. Lichtenstein, A. N. Rubtsov, M. Troyer, and P. Werner, Rev. Mod. Phys. 83, 349 (2011).

[36] P. Werner and A. J. Millis, Phys. Rev. B 74, 155107 (2006).

[37] N. Parragh, A. Toschi, K. Held, and G. Sangiovanni, Phys. Rev. B 86, 155158 (2012).

[38] G. Rushbrooke and P. Wood, Mol. Phys. 6, 409 (1963).

[39] J. Oitmaa and W. Zheng, J. Phys.: Condens. Matter 16, 8653 (2004).

[40] It is important to do a calculation for at least two layers. For the case considered the transition temperature increases by about a factor of 2 when going from one to two layers. Increasing the number of layers beyond two does not increase the transition temperature further.
[41] R. E. Prange and V. Korenman, Phys. Rev. B 19, 4691 (1979).

[42] C. S. Wang, R. E. Prange, and V. Korenman, Phys. Rev. B 25, 5766 (1982).

[43] A. Liechtenstein, M. Katsnelson, V. Antropov, and V. Gubanov, J. Magn. Magn. Mater. 67, 65 (1987).

[44] P. Bruno, Phys. Rev. Lett. 90, 087205 (2003).

[45] A. V. Ruban, S. Shallcross, S. I. Simak, and H. L. Skriver, Phys. Rev. B 70, 125115 (2004).

[46] M. I. Katsnelson and A. I. Lichtenstein, Phys. Rev. B 61, 8906 (2000).

[47] X. Wan, Q. Yin, and S. Y. Savrasov, Phys. Rev. Lett. 97, 266403 (2006).

[48] L. V. Pourovskii, Phys. Rev. B 94, 115117 (2016).

[49] J. Hubbard, Proc. Roy. Soc. London A 276, 238 (1963).

[50] K. S. D. Beach, arXiv:cond-mat/0403055.

[51] J. Mravlje, M. Aichhorn, T. Miyake, K. Haule, G. Kotliar, and A. Georges, Phys. Rev. Lett. 106, 096401 (2011).

[52] L. de Jongh and A. Miedema, Adv. Phys. 23, 1 (1974).

[53] P. Fleury and R. Loudon, Phys. Rev. 166, 514 (1968).

[54] For $\mathrm{KNiF}_{3}$ and $\mathrm{K}_{2} \mathrm{NiF}_{4}$, besides nearest-neighbor exchange the next-nearest-neighbor exchange parameters are known and read, respectively, $J^{\prime} / J=0.005$ and 0.01 [52], leading to no significant change in the transition temperature. For other compounds the effects of $J^{\prime}$ are effectively included in $J$ so we used $J^{\prime}=0$ in these calculations too.

[55] A. Akande and S. Sanvito, J. Chem. Phys. 127, 034112 (2007).

[56] J.-C. Bouloux, J.-L. Soubeyroux, G. L. Flem, and P. Hagenguller, J. Solid State Chem. 38, 34 (1981).

[57] A significant overestimation of the intersite exchange within an approach based on Hubbard-I has been pointed out in Ref. [47]. Apparently, neglecting hybridization effects in the calculation of the impurity Green's function is a rather crude approximation even for strongly localized Mott insulators.

[58] Calculations give, for two nearest neighbors in the $x$ direction, $J_{x}=34.60, J_{y}=34.68$, and $J_{z}=34.68 \mathrm{meV}$; for the $y$ direction the values of $J_{x}$ and $J_{y}$ are interchanged. Hence for the average in-plane $J$ one obtains $34.64 \mathrm{meV}$.

[59] P. A. Serena, N. Garcia, and A. Levanyuk, Phys. Rev. B 47, 5027 (1993).

[60] M. Kastner, R. Birgeneau, G. Shirane, and Y. Endoh, Rev. Mod. Phys. 70, 897 (1998).

[61] C. I. Hiley, M. R. Lees, J. M. Fisher, D. Thompsett, S. Agrestini, R. I. Smith, and R. I. Walton, Angew. Chem. 126, 4512 (2014).

[62] H. Seki, R. Yamada, T. Saito, B. J. Kennedy, and Y. Shimakawa, Inorg. Chem. 53, 4579 (2014). 